\title{
Interference control in cognitive wireless networks by tuning the carrier sensing threshold
}

\author{
Byungjin Cho, Konstantinos Koufos, and Riku Jäntti, \\ Aalto University, School of Electrical Engineering, Espoo, Finland \\ Byungjin.cho@aalto.fi, Konstantinos.koufos@aalto.fi, Riku.jantti@aalto.fi
}

\begin{abstract}
The requirement for secondary spectrum usage is the control of secondary generated interference to the primary system. The generated interference from different types of secondary networks can be controlled by adjusting different parameters. The carrier sensing threshold can be used as a common parameter to control the density of active secondary users in wireless networks with contention control thereby enabling primary system protection. In this paper, we propose a method to set the carrier sensing threshold in secondary wireless networks with finite user density and finite deployment area. Based on the secondary user density, we first find the required distance separation among secondary transmitters so that the primary system service is protected. Given the distance separation, we set the carrier sensing threshold by computing the self-interference at a secondary user. Our method is illustrated for Matern type II and Matern type III point processes for modelling the locations of active secondary users due to the resemblance of these processes to CSMA/CA type of contention control.
\end{abstract}

\section{INTRODUCTION}

A secondary spectrum using system is allowed to access the primary spectrum under the requirement that the quality of the primary system service remains satisfactory. The quality of the primary service can be maintained if the generated secondary interference is controlled under specific protection limits. Recently, the geo-location database has been proposed as a method to coordinate secondary spectrum access [1]. Based on the protection criteria of the primary system, the geo-location database can control some operational parameters of secondary system, enabling interference control.

Cellular and WiFi systems have been proposed as main candidates for secondary spectrum access [2]. Their generated interference to the primary system can be controlled by adjusting different parameters. For a cellular system, the reuse distance can be adjusted to control the number of simultaneous secondary transmissions. For WiFi systems with random access, adjusting the activity factor could be a way to manage the generated interference. For fixed and known locations as in cellular downlink and for Aloha random access, there are widely accepted approximations for the distribution of aggregate interference in slow fading, see for instance [3], [4].

Without contention control, nodes close to each other can transmit simultaneously. This behaviour may be undesirable because the interference generated between nearby nodes may degrade the transmission capacity in a wireless network. Capacity enhancement can be achieved by inhibiting nearby transmissions [5]. This is accomplished by controlling the minimum distance separation among active nodes.

The density of secondary transmitters is a key factor to determining the interference generated to the primary system. As a result, secondary contention control can also be used as a method to control the generated secondary interference [6]. The quality of the primary system service can be maintained through proper selection of the minimum separation distance among the secondary transmitters.

The set of active nodes in networks with contention control is conventionally modelled by a Matern hard-core point process (MPP) [7]-[10]. A MPP is obtained by thinning a Poisson point process (PPP). There are different types of MPPs distinguished based on the rule that governs the selection of effective points i.e. the points that survive the thinning of the PPP. The probability that a point of the PPP is retained and becomes effective is highest for MPP type III and lowest for MPP type I [11]. While the retaining probability in MPP type I and MPP type II is available in closed-form, no results exist so far for the retaining probability in MPP type III [9].

The hardcore distance (HCD) of a MPP essentially models the carrier sensing $(\mathrm{CS})$ range. In practical networks, the CS range is controlled by tuning the $\mathrm{CS}$ threshold. In the existing literature, one can find algorithms for setting the CS threshold within a single network, see for instance [12] for a method to tune the CS threshold in order to achieve a balance between spatial reuse and datarate. Implementing interference control in a primary-secondary system setup by tuning the CS threshold has not received much attention in the existing literature. In [6] the impact of CS range on the interference generated to the primary system is identified. However, there is no proposed algorithm neither for setting the CS range so that the primary system is safely protected nor for mapping the CS range to a CS threshold.

In this paper, we propose a method in order to set the CS threshold in a cognitive radio network with finite user density, finite deployment area and contention control. The MPP type II and MPP type III are used to model the locations of secondary transmitters due to their resemblance to CSMA/CA type of contention control in wireless networks. In order to protect the TV system, we identify an upper bound for the density of secondary transmitters by setting appropriately the HCD. Given the HCD, the CS threshold is presented as a common parameter to control the activity of the secondary network. The proposed method has low complexity and makes it possible to 


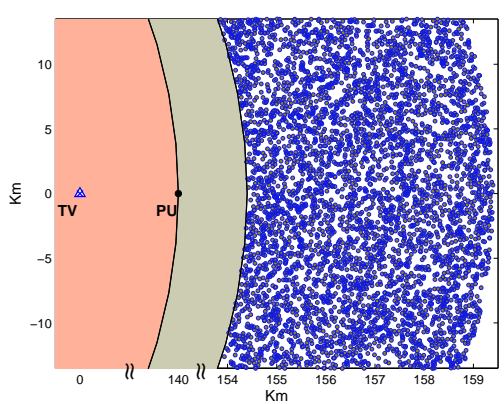

(a)

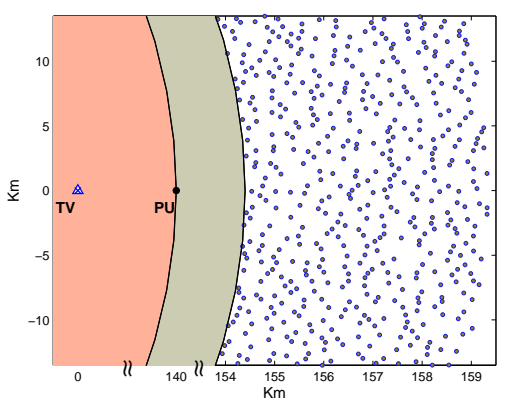

(b)

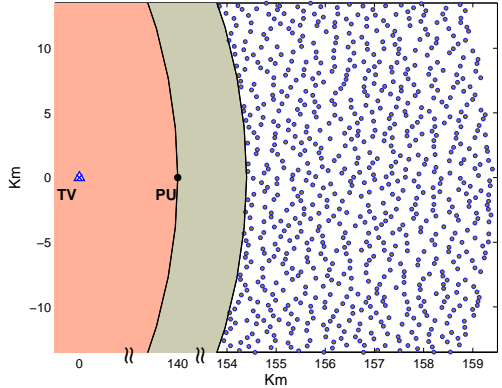

(c)

Fig. 1. System model. A WiFi network operating co-channel to a TV transmitter and deployed in finite area outside of the TV protection area. A snapshot of the point process induced by (a) PPP, (b) MPP type II and (c) MPP type III, with PPP density $\lambda_{p}=100$ users $/ \mathrm{km}^{2}$ and Matern hardcore distance $\delta=300 \mathrm{~m}$.

compute the CS threshold in real-time. As a result, the method can be utilized in geo-location assisted secondary spectrum access even in cases there are frequent changes in secondary user density.

\section{SySTEM MODEL}

We consider a TV transmitter located in the center of a circular TV service area and a WiFi network deployed outside of the TV protection area, see Fig. 1. Protection areas in secondary spectrum sharing have been suggested by Federal Communication Committee (FCC) [13]. The WiFi network operates co-channel to the TV transmitter. For satisfactory TV reception a target SINR, $\gamma_{t}$, must be maintained with specific outage probability $O_{t}$ due to slow fading [14].

$$
O_{t} \geq \operatorname{Pr}\left(\gamma \leq \gamma_{t}\right)
$$

where $\gamma$ is the SINR at the TV receiver $\gamma=\frac{M_{T V}}{I_{S U}+P_{N}}, M_{T V}$ is the received TV signal level, $P_{N}$ is the noise level and $I_{S U}$ is the aggregate interference due to secondary transmissions.

The secondary interference at a TV receiver (PU) located at the TV cell border, see Fig. 1, can be expressed as

$$
I_{S U}=\sum_{k} v_{k} \cdot P_{t} \cdot g_{k} \cdot x_{k}
$$

where $v_{k}$ is a binary factor determining whether the $k$-th secondary user (SU) is active or not, $P_{t}$ is the transmit power level, $g_{k}$ is the attenuation from the $k$-th SU to the PU, and $x_{k}$ is a random variable (RV) describing the slow fading.

The secondary spectrum access can be granted if the required condition for satisfactory TV reception, see equation (1), is satisfied. Equation (1) is a chance type of constraint which is in general difficult to handle. It has been expressed in closed-form in [3] assuming that both the useful TV signal and the aggregate secondary interference follow the log-normal distribution. Under these assumptions the necessary condition for interference control can be turned to the following constraint

$$
\mathrm{E}\left\{I_{S U}\right\} \leq I_{\triangle}
$$

where the interference margin $I_{\triangle}$ determines the maximum permitted mean secondary interference at the PU.
In general, the interference margin depends on the locations of secondary transmitters [3]. However, one has to notice that the secondary generated interference is usually an order of magnitude less than the TV signal level. This fact provides the approximation tightness for the lower bound of the interference margin illustrated in [15], $I_{\triangle l} \leq I_{\triangle}$, which is independent of the SU locations

$$
I_{\triangle l}=\exp \left(\frac{\sigma_{T V}}{\xi} \mathrm{Q}^{-1}\left(1-O_{t}\right)-\ln \left(\gamma_{t}\right)+\frac{m_{T V}}{\xi}\right)-P_{N}
$$

where $\xi=10 / \ln (10), \mathrm{Q}^{-1}$ is the inverse of the Gaussian $Q$ function, $\sigma_{T V}$ in $\mathrm{dB}$ is the standard deviation of the TV signal and $m_{T V}$ in $\mathrm{dB}$ is the $\mathrm{TV}$ signal level at the $\mathrm{PU}$ by using distance-based path loss.

Under contention control, each SU senses the spectrum before its transmission. If the channel is reported busy, the SU postpones its transmission until the channel becomes clear. This behaviour introduces an exclusion region around each SU where no other simultaneous transmissions can take place. The field of transmitters under contention control can be modelled as a hardcore point process, introduced by Matern in [8], since the hardcore process conditions on having a minimum separation distance among the points of the process.

In particular, the MPP type II captures the fact that a SU refrains from transmitting when it senses the activity of another SU which has extracted a smaller back-off time. This behaviour is modelled in the following way: Each point of the process has a random associated mark and a point is discarded only if there is another point within a $\operatorname{HCD} \delta$ with a smaller mark. The density of the MPP II process given the parent density $\lambda_{p}$ and the $\operatorname{HCD} \delta$ is $\lambda_{m}=\frac{1-\exp \left(-\lambda_{p} \pi \delta^{2}\right)}{\pi \delta^{2}}$ [8].

\section{HOW TO SET CS THRESHOLD}

The primary system is protected by maintaining the mean secondary interference under the interference margin $I_{\triangle l}$. The mean interference depends on the active SU density which can be controlled through the HCD $\delta$. In practical networks, the HCD is adjusted by tuning the CS threshold. Mapping the HCD to a CS threshold is not straightforward. While the retaining probability [8] in a MPP is determined by the HCD, 


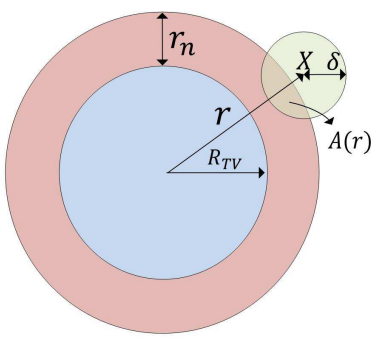

(a)

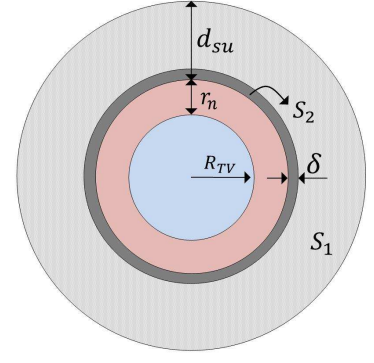

(b)
Fig. 2. Illustration of (a) border effect and (b) two disjoint regions in the secondary deployment area.

the retaining probability in a wireless network depends on the aggregate interference measured at a SU. First, we show how to set the HCD in MPP type II and MPP type III networks without violating the condition $\mathrm{E}\left\{I_{S U}\right\} \leq I_{\triangle l}$. Then, we show how to map the identified HCD to a CS threshold.

\section{A. How to set hardcore distance in MPP type II}

A common way to calculate the mean interference from a MPP type II at an arbitrary location in the plane is to use an equi-dense PPP [16]. In our system setup the deployment area is finite and the active node density close to the borders is higher than $\lambda_{m}$ due to less contention. As a result, setting the HCD based on a homogeneous PPP with density $\lambda_{m}$ will violate the protection criteria at the PU. The PPP approximation worsens for increasing parent density $\lambda_{p}$ and increasing HCD.

With reference to Fig. 2(a), a MPP at distances $r>R_{T V}+$ $r_{n}+\delta$ generates at the PU mean interference equal to the interference generated from a PPP with density $\lambda_{m}$, see also the Appendix for a proof.

In order to compute the generated interference at the PU due to a MPP type II at distances $r:\left(R_{T V}+r_{n}\right) \leq r<$ $\left(R_{T V}+r_{n}+\delta\right)$ we need to compute the density of the process in that area. The density can be found by computing the size of the ball $B(X(r), \delta)$ (with radius $\delta$ and centered at point $X$ at distance $r$ from the TV transmitter) that falls outside of the TV protection area. By looking at the simple geometrical dependency in Fig. 2(a), the size of the area is equal to $\pi \delta^{2}-$ $A(r)$ where $A(r)$ is the intersection area of two circles whose centers are at distance $r$. In Appendix we show that the mean interference due to a MPP type II at distances $r:\left(R_{T V}+r_{n}\right) \leq$ $r<\left(R_{T V}+r_{n}+\delta\right)$ can be upper-bounded by using a PPP with density

$$
\lambda_{2}=\frac{1-\exp \left(-\lambda_{p}\left(\pi \delta^{2}-A\left(R_{T V}+r_{n}\right)\right)\right)}{\pi \delta^{2}-A\left(R_{T V}+r_{n}\right)} .
$$

Since $R_{T V}+r_{n} \gg \delta, A\left(R_{T V}+r_{n}\right) \approx \pi \delta^{2} / 2$. The density $\lambda_{2}$ becomes

$$
\lambda_{2} \approx \frac{1-\exp \left(-0.5 \lambda_{p} \pi \delta^{2}\right)}{0.5 \pi \delta^{2}} .
$$

Obviously, $\lambda_{2}>\lambda_{m}$. Based on the above approximation, the secondary deployment area can be divided into two disjoint regions $S_{1}$ and $S_{2}, S=S_{1} \cup S_{2}$, see also Fig. 2(b). The interference generated by each region to the PU is approximated by a PPP. The densities of the PPP in the two regions are $\lambda_{m}$ for $r \geq\left(R_{T V}+r_{n}+\delta\right)$ and $\lambda_{2}$ for $R_{T V}+r_{n} \leq r<R_{T V}+r_{n}+\delta$.

Since the regions $S_{1}$ and $S_{2}$ are disjoint, the mean interference level at the PU can be computed as a sum of the mean interference levels due to transmissions originated from the areas $S_{1}$ and $S_{2}$. With log-normal slow fading, the mean interference can be approximated to be equal to [4]

$$
\mathrm{E}\left\{I_{S U}\right\} \approx \lambda_{m} \cdot P_{t} \cdot e^{\frac{\sigma^{2}}{2 \xi^{2}}} \int_{S_{1}(\delta)} g_{s} \mathrm{~d} s+\lambda_{2} \cdot P_{t} \cdot e^{\frac{\sigma^{2}}{2 \xi^{2}}} \int_{S_{2}(\delta)} g_{s} \mathrm{~d} s
$$

where $g_{s}$ is distance-based path loss from the location $s$ to the $\mathrm{PU}, \sigma$ in $\mathrm{dB}$ is the $\mathrm{SU}$ slow fading standard deviation and the areas $S_{1}, S_{2}$ are functions of the HCD.

Unfortunately, equation (7) does not have a closed-form solution in terms of the HCD $\delta$. However, we can first find a tight lower bound. The lower bound $\delta_{l}$ assumes PPP with density $\lambda_{m}$ inside full area $S$ and forces the inequality $\mathrm{E}\left\{I_{S U}\right\} \leq I_{\triangle l}$ to be tight. The lower bound can be expressed in terms of the principal branch $\mathcal{W}_{0}$ of the Lambert function

$$
\delta_{l}= \begin{cases}0 & \text { if } \lambda_{p} \leq I_{\triangle_{l}}^{\prime}, \\ \sqrt{\frac{1}{\pi I_{\triangle_{l}}^{\prime}}+\frac{1}{\pi \lambda_{p}} \mathcal{W}_{0}\left(-\frac{\lambda_{p}}{I_{\triangle_{l}}^{\prime}} \cdot e^{\left.-\frac{\lambda_{p}}{I_{\triangle_{l}}^{\prime}}\right)}\right.} & \text { if } \lambda_{p}>I_{\triangle_{l}}^{\prime}\end{cases}
$$

where $I_{\triangle_{l}}^{\prime}=I_{\triangle_{l}} /\left(P_{t} \cdot e^{\sigma^{2} / 2 \xi^{2}} \cdot \int_{S} g_{s} \mathrm{~d} s\right)$.

For user densities, $\lambda_{p} \leq I_{\triangle_{l}}^{\prime}$, we do not need a HCD for protecting the TV system, $\delta=0$, and the CS threshold can be set equal to infinity. For user densities, $\lambda_{p}>I_{\triangle_{l}}^{\prime}$, we can first identify the lower bound $\delta_{l}$ using equation (8). Then, we may define discretization step $\triangle \delta$ and increment the HCD $\delta=\delta_{l}+$ $i \cdot \triangle \delta$ in equation (7) with iteration index $i=1,2, \ldots$ until the constraint $\mathrm{E}\left\{I_{S U}\right\} \leq I_{\triangle l}$ is satisfied. The proposed method identifies a tight upper-bound on the HCD in few iterations.

For reducing implementation complexity one can evaluate offline the integral $\int_{S} g_{s} d s$. Actually, it is beneficial to store the integration results from each ring $(r, \phi)=0 \leq \phi \leq 2 \pi$, $\left(R_{T V}+r_{n}+(j-1) \triangle \delta\right) \leq r \leq\left(R_{T V}+r_{n}+j \cdot \Delta \delta\right), j=$ $1, \ldots d_{s u} / \triangle \delta$. In this way the integrations are not repeated at each iteration and the amount of computations needed to evaluate (7) will be low.

As illustrated in Fig. 3, an equi-dense PPP underestimates the generated interference and results in smaller HCD than the minimum required. The proposed method utilizing superposition of two PPPs can be used as a tight upper bound for the HCD that protects the TV service in all cases.

\section{B. How to set hardcore distance in MPP type III}

In a CSMA/CA type of network, a node that postpones its transmission because it is in the CS range of some other node with lower back-off does not prohibit other nodes to qualify for transmission. MPP type II cannot capture this behaviour because all the nodes are involved in the selection process. Thus, more nodes can survive in a CSMA/CA network compared 


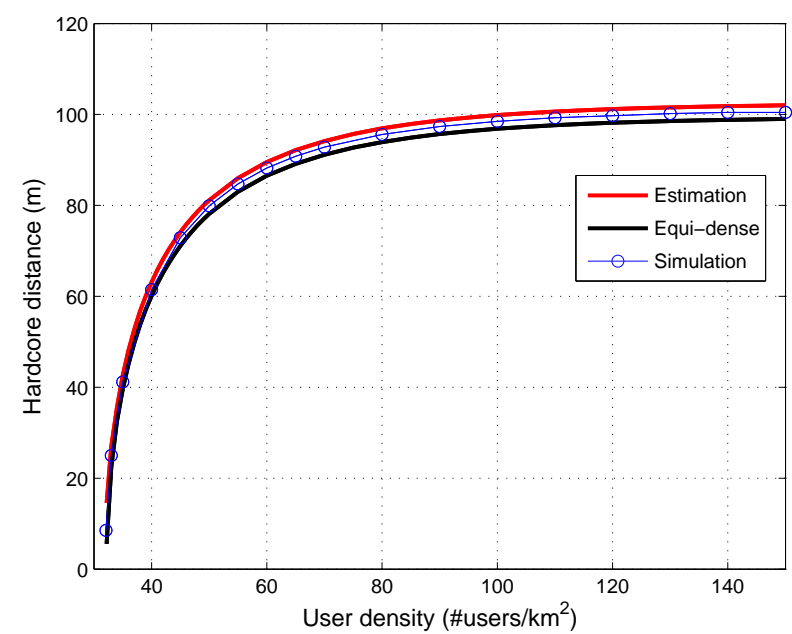

Fig. 3. Hardcore distance for protecting the TV system. The parameter settings can be found in Section IV.

to the number of survived nodes predicted by the MPP type II. In [17] a method to mitigate the underestimation problem of MPP type II is proposed. However, the predicted number of survived points is still less compared with the number of nodes that can survive in a CSMA/CA network. As a result, the protection of the TV service is not secured.

A MPP type III [11] and its temporal variation (SSI) [9] involve only the survived nodes in the selection process. In this sense, these processes model accurately the density of active users in a CSMA/CA network. Unlike MPP type II, the mean number of active nodes in MPP type III cannot be described in closed-form unless the parent density $\lambda_{p}$ goes to infinity [9]. For finite SU densities we can only look for bounds to the mean number of survived nodes in a MPP type III. Due to the analytical tractability of MPP type II, we need to upper bound the number of points generated from a MPP type III by using a MPP type II. A tight bound is difficult to derive because MPP type III is complex to analyze. One simple but loose upper bound suggests to double the HCD of the MPP type II [11].

\section{How to set CS threshold given hardcore distance}

A large HCD can be implemented by setting a low CS threshold and vice versa. In order to map the HCD to a CS threshold we need to compute the mean self-interference at a node of the MPP type II. This is done by using the second moment measure. The final expression involves integrals of the retaining probability which do not accept a closed-form.

In order to overcome this problem we are looking for a lower bound to the mean interference. Note that a lower bound on the CS threshold is in the favour of primary system since it reduces the density of simultaneous transmissions. A lower bound on the mean interference, $\mathrm{E}_{o}^{!}\left\{I_{S U}\right\}$, at a node in the infinite plane has been proposed in [16]. The bound integrates a PPP with density $\lambda_{m}$ in the interval $[\delta, \infty]$. While the proposed bound is tight for any value of HCD and user density, we cannot directly use it because it considers infinite deployment area.

In our system model the mean interference is different at different locations due to the existence of borders. In order to use a common CS threshold, we propose to set it for a node located at the primary protection area border. In this way the CS threshold is underestimated and the TV system is further secured. Similar to [16] we integrate an equi-dense PPP over the secondary area after excluding a ball of radius $\delta$ that is centered at the protection area border. We consider PPP with density $\lambda_{m}$ inside the full area. In this way, the CS threshold is slightly underestimated but the implementation complexity is reduced. Without considering the impact of fading within the secondary network, the CS threshold can be computed as

$$
\mathrm{E}_{o}^{!}\left\{I_{S U}\right\}=\lambda_{m} \cdot P_{t} \cdot \int_{S^{\prime}} g_{s} \mathrm{~d} s
$$

where $S^{\prime}=S \backslash B\left(X\left(R_{T V}+r_{n}\right), \delta\right)$ and $g_{s}$ is the distancebased pathloss from the integration element of area $S^{\prime}$ to the protection area border. For a possible algorithm implementation see the pseudocode below.

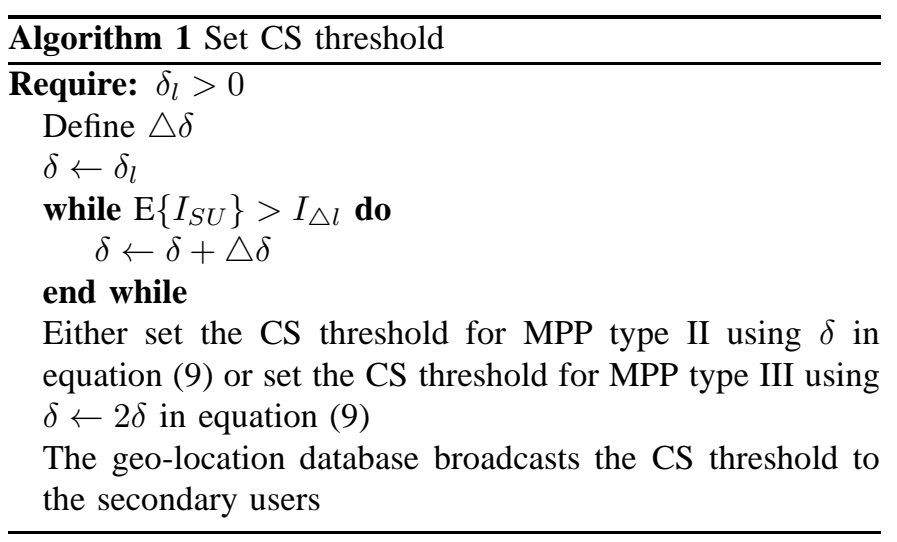

\section{NUMERICAL ILLUSTRATION}

We consider a TV service area with radius $R=140 \mathrm{~km}$ and co-channel protection distance $r_{n}=14.4 \mathrm{~km} \mathrm{[13].} \mathrm{The}$ TV transmitter broadcasts at $P=300 \mathrm{~kW}$. The thermal noise power is $-106.2 \mathrm{dBm}$. We use a power law model for the distance-based propagation pathloss. The path loss exponent for primary and secondary signal is equal to $\alpha_{P U}=3.2$ and $\alpha_{S U}=4$ respectively. The attenuation constant is equal to one. The standard deviations for the log-normal fading distributions are selected equal to $\sigma_{T V}=6 \mathrm{~dB}$ and $\sigma=8 \mathrm{~dB}$. Assuming target SINR $\gamma_{t}=16.1 \mathrm{~dB}$ and target outage probability $O_{t}=10 \%$ the interference margin is calculated by using (4), $-107.3 \mathrm{dBm}$. Outside of the TV protection area the secondary network is deployed. The deployment area is a doughnut with diameter $d_{s u}=5 \mathrm{~km}$, see Fig 2(a). The secondary interference must be controlled at the primary receiver located at PU.

In Fig. 4(a) we depict the CS threshold for different secondary user densities $\lambda_{p}$. First, we simulate the CS threshold for the MPP type III. In the simulations, we find iteratively the maximum CS threshold that protects the TV service. We 


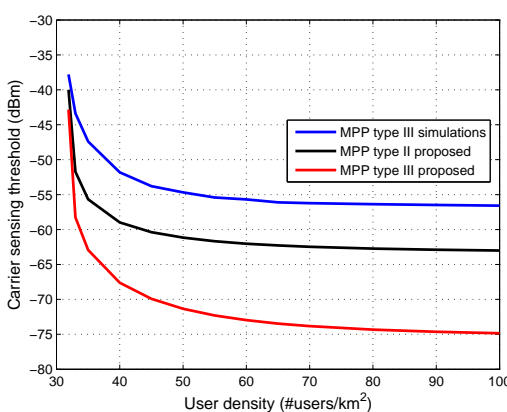

(a)

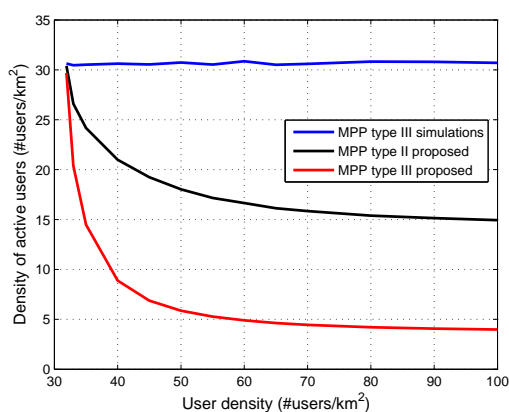

(b)

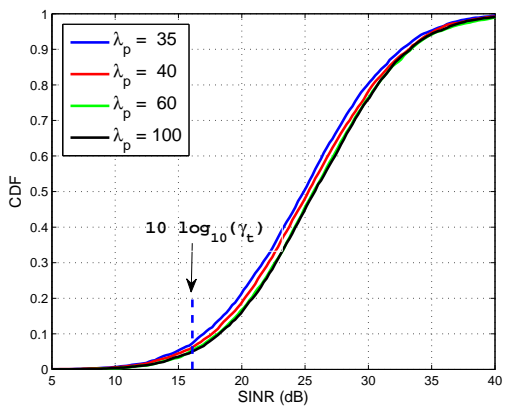

(c)

Fig. 4. (a) CS threshold set by the simulation, the HCD in MPP type II and the proposed method (b) Active density and (c) SINR distribution at TV receiver

define discretization step equal to $0.1 \mathrm{~dB}$ and increment the CS threshold until the constraint $\mathrm{E}\left\{I_{S U}\right\} \leq I_{\triangle l}$ is satisfied. In each iteration we run a Monte Carlo simulation with 1000 trials. The steps carried out in each trial are described below.

We draw the number of secondary users from a Poisson probability distribution function (PDF) with density $\lambda_{p}$. The users are deployed randomly inside the secondary area $S$. Every node is ranked and the node with rank one always transmits. For the $k$-th node we compute the mean interference from the survived nodes upto the $(k-1)$-th node and compare it with the CS threshold. The node transmits only if the mean interference is below the CS threshold. This selection process is a modified version of the SSI model [10]. For the configuration of active nodes we simulate the generated fading samples at the PU and compute the mean aggregate interference. If the constraint $\mathrm{E}\left\{I_{S U}\right\} \leq I_{\triangle l}$ is satisfied in all the simulation trials the CS threshold for the considered user density is stored. Otherwise, the CS threshold is incremented.

One can see that the CS threshold decreases for increasing user density implementing essentially a larger HCD. In Fig. 4(a) we also depict the CS threshold using the proposed method for MPP type II and MPP type III. The proposed method for the MPP type III results in very low CS threshold because the calculated HCD has been doubled, see Section III-B. The proposed method for the MPP type II also results in lower threshold compared with the simulations. This is because we used several approximations for setting conservatively both the HCD and the CS threshold. While setting the HCD we utilized: (i) a lower bound on the interference margin to reduce computational complexity and (ii) an upper bound on the HCD to deal with border effects. Also, the CS threshold: (iii) has been set based on the interference level at the protection area border (iv) has been bounded using the PPP approximation and (v) did assume uniform density of effective secondary users inside the deployment area.

Fig. 4(b) shows the density of active secondary users using the CS threshold depicted in Fig. 4(a). When the simulated thresholds are used, the density of secondary transmitters sustains a mean around 30.5 transmitters $/ \mathrm{km}^{2}$. In our system setup, this is the highest density not violating the TV protection criteria. On the other hand, when the CS threshold is set based on the proposed method, the density of transmitters is quickly reduced. For the MPP type III, the CS threshold is conservatively set because the HCD is doubled. That significantly reduces the retaining probability and the density of active users. When the CS threshold is set based on the MPP type II, the active node density at high user densities is about half the maximum permitted density.

In Fig.4(c) the SINR distribution at the PU is simulated for different secondary user densities. If the simulated thresholds are used, the outage probability at the SINR target would be equal to the outage probability target $10 \%$. The results of Fig.4(c) are generated using the CS threshold for the MPP type II. The target is to illustrate the reduction in outage probability due to the conservative approximations adopted by our proposal. For high user densities, where the active node density is about half the maximum permitted, the outage probability is about $5 \%$.

\section{CONClusion}

In this paper, we proposed a method to set the CS threshold in a geo-location assisted secondary network with finite user density, finite deployment area and contention control. The CS threshold was viewed as a parameter that can be tuned to control the generated secondary interference and maintain satisfactory primary system service. The geo-location database is responsible for calculating the CS threshold and for broadcasting it to the secondary users.

We identified a critical value for the secondary user density below which the CS threshold can be set to infinity. For higher user densities we proposed a low complexity method for setting the CS range and mapping it to a CS threshold. The proposed method for MPP type III sets the CS threshold conservatively. Given our parameter settings, doubling the hardcore distance reduces the CS threshold by $12 \mathrm{~dB}$ in comparison with the threshold calculated based on the MPP type II. In order to enable a higher density of secondary transmitters, we need to identify a tighter upper bound for the number of points survived in a MPP type III.

\section{APPENDIX}

Let us consider $N$ users in the doughnut $S:(r, \phi)=\{0 \leq$ $\left.\phi \leq 2 \pi, R_{\text {in }} \leq r \leq R_{\text {out }}\right\}$ where $R_{\text {in }}=R_{T V}+r_{n}$ and $R_{\text {out }}=$ 
$R_{i n}+d_{s u}$. For simplicity, we set $P_{t}=1$. The mean interference at the PU is

$$
\begin{aligned}
\mathrm{E}\left\{I_{S U}(N)\right\} & =\sum_{i=1}^{N} \int_{R_{\text {in }}}^{R_{\text {out }}} \frac{2 \pi r}{S}\left(\frac{B(r)-\pi \delta^{2}}{S}\right)^{i-1} g(r) \mathrm{dr} \\
& =2 \pi \int_{R_{\text {in }}}^{R_{\text {out }}} \frac{1-\left(\left(B(r)-\pi \delta^{2}\right) / S\right)^{N}}{S+\pi \delta^{2}-B(r)} r g(r) \mathrm{dr}(10)
\end{aligned}
$$

where $S=\pi\left(R_{\text {out }}^{2}-R_{\text {in }}^{2}\right)$ is the area of the doughnut and $B(r)$ is the union of the doughnut and of a circle with radius $\delta$.

Since the users are uniformly distributed, the term $2 \pi r / S$ describes the probability of distributing a user at distance $r$ from the inner radius $R_{i n}$. Also, the term $\left(\left(B(r)-\pi \delta^{2}\right) / S\right)^{i-1}$ describes the probability that the $i$-th user becomes active. Particularly, the term $\left(\left(B(r)-\pi \delta^{2}\right) / S\right)$ equals the probability that a point located at distance $r$ from the inner radius is not covered when a disc of radius $\delta$ is randomly thrown. Since the centers of the discs are uniformly and independently thrown, a point is not covered with probability $\left(\left(B(r)-\pi \delta^{2}\right) / S\right)^{j}$ after $j$ discs have been thrown.

Case 1: $(r, \phi)=\left\{0 \leq \phi \leq 2 \pi, R_{\text {in }}+\delta \leq r \leq R_{\text {out }}\right\}$. In that case, $B(r)=S$. After replacing $B(r)$ in equation (10) we get

$$
\mathrm{E}\left\{I_{S U}(N)\right\}=\frac{2 \pi}{\pi \delta^{2}}\left(1-\left(1-\pi \delta^{2} / S\right)^{N}\right) \int_{R_{\text {in }}}^{R_{\text {out }}} r g(r) \mathrm{dr} .
$$

The average number of users follows Poisson distribution with mean $\lambda_{p} S$. The mean interference at the PU can be computed by weighting the mean generated interference due to $k$ users, $\mathrm{E}\left\{I_{S U}(k)\right\}$, with the Poisson PDF.

$$
\begin{aligned}
\mathrm{E}\left\{I_{S U}\right\} & =\sum_{k=0}^{\infty} \mathrm{E}\left\{I_{S U}(k)\right\} \cdot \frac{\left(\lambda_{p} S\right)^{k} \cdot \mathrm{e}^{-\lambda_{p} S}}{k !} \\
& =\frac{2 \pi \cdot\left(1-\mathrm{e}^{-\lambda_{p} \pi \delta^{2}}\right)}{\pi \delta^{2}} \int_{R_{\text {in }}+\delta}^{R_{\text {out }}} r g(r) \mathrm{dr} .
\end{aligned}
$$

The right hand side of equation (12) is the mean interference due to a PPP with density $\lambda_{m}=\left(1-\mathrm{e}^{-\lambda_{p} \pi \delta^{2}}\right) / \pi \delta^{2}$. As a result, a MPP type II in the area $(r, \phi)=\{0 \leq \phi \leq 2 \pi$, $\left.R_{\text {in }}+\delta \leq r \leq R_{\text {out }}\right\}$ generates mean interference at the PU equal to the mean interference generated by an equi-dense PPP. The impact of outer border has been ignored in the analysis but actually, it can be treated similar way to case 2 .

Case 2: $(r, \phi)=\left\{0 \leq \phi \leq 2 \pi, R_{\text {in }} \leq r \leq R_{\text {in }}+\delta\right\}$. In that case, $B(r) \leq S+A\left(R_{i n}\right)$. Let us consider the function $f(x)=\frac{1-\left(\left(x-\pi \delta^{2}\right) / S\right)^{N}}{S+\pi \delta^{2}-x}$. The function $f$ can also be read as $f(u)=S^{-1} \cdot \sum_{n=0}^{N-1} u^{n}, u=\left(x-\pi \delta^{2}\right) / S, 0<u<1$. The function $f(u)$ is increasing in $u$ and thus, $f(x)$ is increasing in $x$. As a result, one can upper-bound the mean interference by setting $B(r)=S+A\left(R_{\text {in }}\right)$ in equation (10)

$$
\mathrm{E}\left\{I_{S U}(N)\right\}<2 \pi \int_{R_{i n}}^{R_{i n}+\delta} \frac{S^{N}-\left(S+A\left(R_{i n}\right)-\pi \delta^{2}\right)^{N}}{S^{N}\left(\pi \delta^{2}-A\left(R_{i n}\right)\right)} r g(r) \operatorname{dr} .(13)
$$

By following same approach as in equation (12) we get

$$
\mathrm{E}\left\{I_{S U}\right\}<\frac{2 \pi\left(1-\mathrm{e}^{-\lambda_{p}\left(\pi \delta^{2}-A\left(R_{i n}\right)\right)}\right)}{\pi \delta^{2}-A\left(R_{i n}\right)} \int_{R_{i n}}^{R_{i n}+\delta} r g(r) \mathrm{dr} .
$$

As a result, the mean interference generated by a MPP type II in the doughnut $(r, \phi)=\left\{0 \leq \phi \leq 2 \pi, R_{\text {in }} \leq r \leq R_{\text {in }}+\delta\right\}$ can be upper-bounded by computing the mean interference due to a PPP with density $\left(1-\mathrm{e}^{-\lambda_{p}\left(\pi \delta^{2}-A\left(R_{i n}\right)\right)}\right) /\left(\pi \delta^{2}-A\left(R_{i n}\right)\right)$.

\section{ACKNOWLEDGMENT}

This work was partially supported by the TEKES funded project IMANET+ and by the Academy of Finland funded project SMACIW under grant number 265040.

\section{REFERENCES}

[1] Ofcom, "Implementing geolocation", Consultation, Nov. 2010.

[2] J. Kronander, M. Nekovee, K.W. Sung, J. Zander, S.L. Kim, and A. Achtzehn, "QUASAR scenarios for white space assessments and exploitation", in Proc. Conf. URSI EMC, 2010.

[3] K. Ruttik, K. Koufos, and R. Jäntti, "Modeling of the secondary systems generated interference", J. Radioengineering, vol. 10, no. 4, pp. 488-493, 2010.

[4] K. Koufos, K. Ruttik, and R. Jäntti, "Aggregate interference from WLAN in the TV white space by using terrain-based channel model", in Proc. IEEE Crowncom, Jun. 2012.

[5] A. Hasan and J. G. Andrews, "The guard zone in wireless ad hoc networks", IEEE Trans. Wir. Commun., vol. 6, pp. 897-906, 2007.

[6] Z. Chen, C. Wang, X. Hong, J. Thompson, S. A. Vorobyov, X. Ge, H Xiao, and F. Zhao, "Aggregate interference modeling in cognitive radio networks with power and contention control", IEEE Trans. Commun., vol. 60, no. 2, pp. 456-468, 2012.

[7] M. Haenggi and R. K. Ganti, "Interference in large wireless networks", Found. Trends Netw., vol. 3, no. 2, pp. 127-248, 2009.

[8] Matèrn, B., 1960. Spatial variation. stochastic models and their application to some problems in forest surveys and other sampling investigations. Medd. Statens Skogsforskningsinstitut, 49 (5), 1-144.

[9] A. Busson, C. Chelius, and J. M. Gorce, "Interference modeling in CSMA multi-hop wireless networks", INRIA Tech. Rep. 6624, 2009.

[10] A. Busson and G. Chelius, "Point processes for interference modeling in CSMA/CA ad-hoc networks", in Proc. ACM PE-WASUN, 2009.

[11] J. Møller, M. L. Huber, and R. L. Wolpert, "Perfect simulation and moment properties for the Matèrn type III process", Stochastic Processes and their Applications, vol. 120, no. 11, pp. 2142-2158, 2010.

[12] T.S. Kim, H. Lim and J.C. Hou, "Improving spatial reuse through tuning transmit power, carrier sense threshold, and data rate in multihop wireless networks", in Proc. MobiCom, 2006.

[13] In the matter of unlicensed operation in the TV broadcast bands: Third memorandum opinion and order, Federal Communications Commission, FCC 12-36A1, 2012, http://hraunfoss.fcc.gov/edocs_public/ attachmatch/FCC-12-36-A1.pdf.

[14] U. Reimers, DVB-the family of international standards for digital video broadcasting, Springer, Berlin, Germany, 2nd edition, 2005.

[15] K. Koufos, K. Ruttik, and R. Jäntti, "Controlling the interference from multiple secondary systems at the TV cell border", in Proc. IEEE PIMRC, 2011.

[16] M. Haenggi, "Mean interference in hard-core wireless networks", IEEE Commun. Lett., vol. 15, no. 8, pp. 792794, 2011.

[17] H. ElSawy, E. Hossain and S. Camorlinga, "Characterizing random CSMA wireless networks: A stochastic geometry approach", in Proc. IEEE ICC, 2012.

[18] H. Q. Nguyen, F. Baccelli, and D. Kofman, "A Stochastic Geometry Analysis of Dense IEEE 802.11 Networks", in Proc. IEEE INFOCOM, 2007. 